\title{
Paul Claval, Penser le monde en Géographe
}

Paris : l'Harmattan, 268 pages.

\section{Michaël Picon}

\section{(2) OpenEdition \\ Journals}

Electronic version

URL: http://journals.openedition.org/rge/5690

ISSN: 2108-6478

\section{Publisher}

Association des géographes de l'Est

\section{Printed version}

Date of publication: 22 April 2016

ISSN: 0035-3213

\section{Electronic reference}

Michaël Picon, « Paul Claval, Penser le monde en Géographe », Revue Géographique de l'Est [Online], vol. $56 / n^{\circ} 1-2$ | 2016, Online since 25 August 2016, connection on 22 September 2020. URL : http:// journals.openedition.org/rge/5690

This text was automatically generated on 22 September 2020

Tous droits réservés 


\section{Paul Claval, Penser le monde en Géographe}

Paris : l'Harmattan, 268 pages.

Michaël Picon

\section{REFERENCES}

Paul Claval, Penser le monde en Géographe, l'Harmattan, 268 p. 
Voici un ouvrage bien précieux pour le géographe. Il apparaît en effet comme le point nodal de la vaste toile géographique tissée par Paul Claval pendant soixante ans. Sans être son propre thuriféraire, P. Claval dresse, selon un plan essentiellement chronologique, l'inventaire critique de ses travaux de Géographe. Le livre est organisé en deux grandes parties. La première est rédigée au passé et traite de la «nouvelle Géographie ", la seconde est écrite au présent et traite des domaines que $P$. Claval étudie encore. La préface d'Isabelle Lefort annonce la teneur de l'ouvrage en nous rappelant que l'on a affaire à un grand Géographe et à un grand Épistémologue contemporain. Ce livresynthèse est donc un livre de passeur et de modernisateur. Il ressemble à une vaste épistémologie de la Géographie humaine, essentiellement contemporaine, car P. Claval a participé et participe encore à celle-ci avec ferveur.

L'auteur explique l'évolution de ses travaux des années 1950 à aujourd'hui après avoir donné les grands principes constants qui dirigent sa pensée de géographe. Ils sont peu nombreux car, pour lui, l'univers idéel est un océan mouvementé qui ne peut être contraint. Ses objectifs sont clairs, il cherche sans cesse à faire l'aggiornamento de la Géographie humaine en lui insufflant les idées d'autres disciplines. Ce sera d'abord l'Économie (essentiellement des années 1950 aux années 1970). En parallèle, P. Claval s'intéresse (à partir de 1965) à la Sociologie et à l'Ethnologie, il regarde aussi vers la Philosophie et l'Anthropologie politique (à partir des années 1970). Ses lectures scientifiques sont riches et, a minima, bilingues (français et anglais). Ses sens de Géographe lui permettent d'extraire l'essence géographique de chacune des matières qu'il étudie. Il fait passer dans le champ géographique des idées économiques telles que les économies d'échelle, les économies externes, les portées-limites, le schéma de Fourastié et la théorie des lieux centraux, etc.

3 Il exploite les ressources intellectuelles de la sociologie et de l'anthropologie lorsqu'il s'aperçoit que la géographie économique, notamment le fameux schéma triangulaire d'Alfred Weber ${ }^{1}$, ne suffit plus à expliquer l'implantation des industries du XXe siècle ${ }^{2}$. Sous l'influence des travaux de l'ethnologue Ralph Linton ${ }^{3}$, P. Claval ajoute une dimension sociale à sa Géographie, conscient que la dimension économique de la Géographie humaine ne suffit pas pour la moderniser. Il fait un constat majeur : la Géographie humaine ne tient pas assez compte des relations entre individus. Il suggère que les relations sociales sont, spatialement, perçues comme « distances» ou « circulations ». C'est l'intégration de l'espace perçu comme distance à la Géographie humaine qui fait de cette dernière une Géographie sociale... si l'on ne considère pas la 
distance comme une simple métrique, mais comme ce qui facilite ou restreint les relations (ici sociales) dans l'espace.

4 Après avoir constaté que la Géographie politique avait été, surtout en France, discréditée par ses liens avec le fascisme et le national-socialisme, P. Claval évoque sa renaissance et les influences de la nouvelle Géographie politique. Pour lui, renouveler la Géographie politique consistait à élargir son approche traditionnelle ${ }^{4}$ en considérant que l'État intégrait désormais la notion de liberté ${ }^{5}$ en plus des potestat et autorictas ${ }^{6}$ médiévaux. Il s'agit donc d'ajouter un thème majeur (la liberté) aux deux thèmes traditionnels de la Géographie Politique. Ainsi, P. Claval indique que sa Géographie politique nouvelle s'inspirait à la fois des philosophes politiques traditionnels (de Machiavel à Rousseau) et de penseurs plus contemporains ${ }^{7}$. Elle s'appuie sur les quatre relations wébériennes de pouvoir: le pouvoir pur, l'autorité, l'influence et la domination. Il constate que les deux premières ont des implications spatiales fortes dans les systèmes politiques anciens. La première, la relation de pouvoir pur, nécessite une surveillance forte du territoire, et donc une bonne organisation administrative territoriale. La seconde, la relation d'autorité, nécessite d'avoir une culture homogène voire commune dans le territoire du système politique concerné. Enfin, les deux nécessitent d'assurer la sécurité extérieure par différents moyens: armée, communications, ravitaillement. Les systèmes politiques modernes ont subi l'influence d'une mutation née de la Réforme : la souveraineté (autorictas) provient des citoyens. P. Claval distingue alors deux systèmes politiques modernes liés à cette première mutation: les systèmes libéraux, dans lesquels l'autorité provient de l'opinion publique, elle-même influencée par les intellectuels; les systèmes totalitaires, dans lesquels l'autorité provient d'une idéologie imposée par le musellement de l'opinion publique. P. Claval consacre aussi deux ouvrages à la Géopolitique et aux relations internationales : Espace et pouvoir (1978) et Géopolitique et géostratégie (1992). Le premier traitait essentiellement des relations entre les acteurs de la scène internationale. Selon lui, ces relations étaient liées à trois processus : la violence et la menace, les questions de distance (accès aux ressources et aux informations, mouvements militaires) et la culture (et sa prise en compte dans les relations internationales). Dans le second, l'ouvrage de 1992, il étendait sa réflexion à la Géopolitique ${ }^{8}$. Il la ressent surtout comme une polémo-Géographie, auparavant (en France) vouée au développement de la puissance du Pays, puis vouée à l'établissement d'un équilibre lorsque l'on admet que le Pays est devenu une puissance de second plan. Il constate que pendant la guerre froide, la Géopolitique des grands blocs se mue en une Géostratégie d'un genre nouveau. Celleci était fondée sur la course à l'armement, alors que dans les Pays où la guerre froide était absente, la Géopolitique plus traditionnelle se maintenait. La fin de la guerre froide nous ramène à une forme classique de Géopolitique, avec les enjeux de notre époque en sus. En somme, il ajoute à sa Géographie Politique les questions de distance et de culture9. Ses travaux sur les Géographies économique, humaine et politique annoncent d'une certaine manière la Géographie culturelle qu'il portera dans la seconde partie de sa carrière.

5 Sa Géographie urbaine est fortement inspirée d'auteurs allemands ${ }^{10}$ et étasuniens ${ }^{11}$. Dans les années 1970, il complète la théorie des lieux centraux (d'inspiration économique) par le concept de coût de commutation. L'idée lui est inspirée par les télécommunications, il la décrit dans un colloque sur les transports à Dijon le 15 mars 1976. Cela l'amène à penser, en plus de la hiérarchisation des axes de transports, la hiérarchisation des pôles. Il explique ainsi la suburbanisation et la rurbanisation. Il 
s'intéresse aussi, à une échelle plus grande, à la logique des villes. Cela l'amène à proposer trois principes d'interprétation de la ville et à en déduire son analyse de l'espace urbain. Il considère que la ville est apparue historiquement à cause de la nécessité pour l'Homme de communiquer et de se rassembler, qu'elle appartient à une hiérarchie de lieux centraux et qu'elle facilite trois types de relations (commerce, informations, informations confidentielles). Sa vision de la ville est, en somme, fondée sur une idée principale : la ville est née et s'est organisée pour faciliter tous les types de communications. Les problématiques de distance sont ainsi toujours présentes dans la pensée clavalienne.

6 P. Claval traite aussi de Géographie régionale. Il considère celle-ci comme une approche plutôt que comme un domaine de la Géographie. Celle-ci consiste à définir des régions en fonction d'un certain nombre de critères. Ces régions étaient d'abord définies par des critères naturels ou physiques, puis la méthode s'est améliorée, affinée, et a adopté des critères humains (en particulier grâce à l'apparition de la cartographie thématique). L'approche s'était dans le même temps systématisée et se voulait globale. Dans les deux cas, elle s'inspirait des sciences $«$ dures $»^{12}$. La Géographie humaine acquiert ainsi une image plus scientifique. Plus récemment, Claval intègre la culture et l'identité à cette démarche. On ne parle plus de régions, mais de territoires, d'espaces vécus et appropriés. Cette façon de faire de la Géographie le pousse naturellement vers une Géographie culturelle, tournée vers les Hommes, leurs représentations et leurs cultures.

7 P. Claval développe sa Géographie culturelle dans la seconde partie de sa carrière ${ }^{13}$. Il considère la première étape de ses recherches comme une propédeutique. Selon lui, la Géographie culturelle permet de répondre à certaines interrogations, notamment celles que la Géographie politique pose autour du territoire ${ }^{14}$. P. Claval dresse une épistémologie de la matière. Fin XIX ${ }^{\text {ème }}$ et début $\mathrm{XX}^{\mathrm{ème}}$ siècles, elle est d'abord proche de l'ethnologie, et s'intéresse essentiellement à la culture des peuples historiques et préhistoriques. Elle est alors influencée par les théories évolutionnistes et P. Claval lui reproche de réfuter le rôle des représentations, des symboles, et de ne regarder que les rapports de l'Homme à l'environnement, avec une approche trop déterministe. Il s'intéresse à deux Géographes français qui modernisent la matière : Xavier de Planhol qui étudie dans les années 1950 le monde musulman et intègre le fait religieux dans la compréhension de l'espace; Pierre Gourou qui, dans les années 1970, remplace le déterminisme naturel par un déterminisme de civilisation. Progressivement, sous l'influence de ses lectures et de ses recherches épistémologiques, P. Claval ajoute une dimension culturelle à sa Géographie. Il réajuste réellement ses travaux dans les années 1970, influencé par le concept de topophilie ${ }^{15}$. Il s'associe à Roger Brunet et à l'espace géographique créé par ce dernier en 1972, mais des divergences sur la prévalence ou non de l'économie dans l'organisation de l'espace entraînent une rupture entre R. Brunet et P. Claval dans les années 1980 .

8 P. Claval ne traite pas uniquement de Géographie culturelle dans la seconde moitié de sa carrière, il touche encore à la plupart des Géographies. Ainsi, il synthétise dans son ouvrage sa vision du monde contemporain observé au travers d'un prisme géographique. Il évoque les grandes mutations de celui-ci, qu'elles soient économiques (libéralisation de l'économie), géographiques (réduction des distances par les télécommunications et par l'amélioration des transports, crise de l'urbanité, etc.), culturelles (globalisation et cultures de masse), ou géopolitiques (problème de la 
cohérence des territoires nationaux dans un monde de plus en plus réticulaire). Il pense que la discipline a besoin d'un aggiornamento pour bien analyser le monde actuel, et la construction de celui-ci passe par une bonne connaissance épistémologique de la Géographie.

Durant sa carrière, P. Claval étudie par exemple les mythes fondateurs de la pensée occidentale en matière de sciences sociales ${ }^{16}$ et leurs implications spatiales. Ses recherches l'amènent à étudier la pensée occidentale au travers des $\operatorname{arts}^{17}$, des sciences et de la philosophi ${ }^{18}$. Toutes ces influences peuvent expliquer sa nouvelle vision de la Géographie qu'il semble justifier ainsi : "John Paul Jones III et Wolfgang Natter (1999) situent au XVIIIe siècle le moment où se structure la géographie actuelle. La discipline souffre de la coupure qui existe entre les théories de l'espace et celles de la représentation. La division entre espace et représentations, ou entre science et poésie, est une conséquence du rationalisme des Lumières et résulte des jeux du pouvoir [...]» (page 205). Le bilan épistémologique de la discipline l'amène à cette conclusion: il faut systématiquement intégrer les valeurs dont l'Homme charge le paysage à la démarche géographique. Il définit trois grilles de lecture du paysage : une grille fonctionnelle (à quoi sert-il ?), une grille archéologique (le paysage vu comme palimpseste), une grille symbolique (de quelles valeurs, de quels symboles, de quelles émotions est chargé le paysage? Quels sens lui donne-t-on ?). Il développe toujours le substrat épistémologique de son travail et sa démarche est, évidemment, fortement influencée par l'œuvre de P. Vidal de La Blache. Il considère d'ailleurs que cette dernière n'a pas été entièrement prise en compte par ses héritiers. Il voit le concept vidalien de Genre de vie comme une adaptation du déterminisme évolutionniste, comme un « possibilisme vidalien ».

En lisant cet ouvrage, on comprend que l'Épistémologue Claval ne pouvait devenir autre chose que Géographe culturel. En effet, ce grand lecteur ne rejette pas son héritage, il l'acquiert pendant toute sa carrière, le critique et le fait fructifier dans le même temps, et ce pendant au moins soixante ans. Il pratique une Géographie d'accumulation plutôt qu'une Géographie d'exclusion. À sa manière, il met en valeur et revitalise le patrimoine géographique. Ce livre nous permet de suivre et de comprendre le fil de la pensée géographique de P. Claval. C'est un excellent guide pour celui qui cherche des ressources épistémologiques. On peut seulement regretter l'absence de figures dans certains cas. Par exemple, le concept des coûts de commutation, inspiré par les télécommunications à $\mathrm{P}$. Claval, serait plus aisé à comprendre à l'aide d'un croquis. Certes, cela altérerait l'idée, mais P. Claval ne cite-t-il pas lui-même Paul Valéry : «Le simple est toujours faux. Ce qui ne l'est pas est inutilisable »"19 Enfin, l'honnêteté de l'auteur est remarquable, notamment lorsqu'il évoque le manque de visibilité de sa Géographie dû, parfois, à des divergences idéologiques avec ses collègues, lorsqu'il admet que la Géographie physique manque à sa démarche, lorsqu'il raconte modestement qu'un étudiant ne sait pas qui il est, ou lorsqu'il évoque sa rupture avec la géographie brunetienne. Ainsi, les soixante années de pensée clavalienne synthétisées ici forment un pli leibnizien, qui, une fois déplié, contient tout un univers géographique. 


\section{NOTES}

1. Ce schéma situe l'emplacement idéal d'une usine au barycentre de trois points (les matières premières, la source d'énergie et le marché) pondérés selon leur importance.

2. À la fin du XIXe siècle, l'implantation des usines sidérurgiques lorraines subit parfois l'influence des conflits et des tensions avec l'Allemagne. Dès cette époque, le modèle wébérien, qui ne prend pas en compte les éléments géopolitiques, ne fonctionne plus et apparaît comme simpliste en oubliant de nombreux facteurs. À la fin du XXe siècle, les territoires où les conflits sociaux sont récurrents n'incitent pas les usines à s'y implanter; les coûts des transports fortement réduits par la conteneurisation et l'influence de la mondialisation sur le prix des matières premières bouleversent les stratégies de localisation initiées par le modèle wébérien. Ces éléments, parmi d'autres, rendent le schéma particulièrement obsolète aujourd'hui, Claval l'avait remarqué quasiment au moment où celui-ci le devenait.

3. R. Linton (1968), De L'homme, Paris, Minuit, 535 pages. R. Linton étudie essentiellement les liens entre l'homme et son milieu.

4. Selon lui, il s'agit de celle qui était influencée par les philosophies politiques de Machiavel, Hobbes, Locke et Rousseau. Elle se consacre essentiellement à l'étude de l'exercice du pouvoir.

5. L'idée lui vient des travaux de John Rawls (A Theory of Justice, 1971).

6. Le pouvoir et la souveraineté (Dieu, indirectement le Pape) qui permet de garder le contrôle sur le premier. La réforme protestante ajoute une certaine idée de la liberté à ces deux éléments d'après P. Claval. On peut aussi y voir l'influence des humanistes de la Renaissance, et particulièrement celle d'Érasme de Rotterdam.

7. Il cite notamment Michel Foucault et son célèbre Surveiller et punir, Max Weber et Jean Gottman.

8. La Géopolitique traite plutôt du temps court (stratégies par exemple), alors que la Géographie politique traite du temps long (jeux d'équilibre...).

9. Pour P. Claval, la culture c'est tout ce qui n'est pas inné chez l'Homme.

10. La sociologie allemande du XIXe siècle.

11. L'école de Chicago du XXe siècle.

12. La cartographie thématique est imprégnée de statistiques et de mathématiques ; l'approche systémique et globale s'inspire de la Géographie physique (d'abord russe).

13. Elle devient sa discipline majeure dans les années 1980 .

14. Les conceptions traditionnelles du territoire-nation sont de plus en plus désuètes du fait de la globalisation, une définition neuve du territoire est nécessaire.

15. Concept développé par Yi-Fu Tuan dans les années 1970 s'intéressant aux représentations que l'Homme se fait de l'espace.

16. Il évoque les trois contrats sociaux proposés par Hobbes, Locke et Rousseau.

17. Il étudie les chronotopes, les dimensions spatiales et temporelles des œuvres littéraires.

18. Il accumule des notes dans ce domaine, sans avoir le temps d'en tirer un article.

19. In Euvres II (1942), Paul Valéry, éd. Gallimard, coll. Bibliothèque de la Pléiade, 1960, page 864 


\section{AUTHOR}

\section{MICHAËL PICON}

Doctorant en géographie, université de Lorraine 